\title{
A Genetic Algorithm for Predicting RNA Pseudoknot Structures
}

\author{
Dongkyu Lee and Kyungsook Han*
}

School of Computer Science and Engineering, Inha University, Inchon 402-751, Korea

\begin{abstract}
An RNA pseudoknot is a tertiary structure element formed when bases of a single-stranded loop pair with complementary bases outside the loop. Computational determination of the RNA folding structure with pseudoknots from the linear sequence is a complex problem involving both spatial reasoning and the use of knowledge of chemistry and biology. We have developed a genetic algorithm for predicting the RNA folding structure with pseudoknots of any type. This paper analyzes the predictions by the genetic algorithm with different population generation methods and fitness functions, and compares the predictions to those by a dynamic programming method.
\end{abstract}

\section{Introduction}

An RNA pseudoknot is a tertiary structural element formed when bases of a singlestranded loop pair with complementary bases outside the loop. Pseudoknots are not only widely occurring structural motifs in all kinds of viral RNA molecules, but also responsible for several important functions of RNA. The RNA folding structure with pseudoknots is much more difficult to predict than the RNA secondary structure because prediction of pseudoknots should consider tertiary interactions as well as secondary interactions.

In the computational viewpoint, predicting RNA structure can be considered as an optimization problem. Many prediction algorithms obtain the RNA structure with the smallest free energy as an optimal structure [1]. Greedy algorithms and dynamic programming algorithms were also used to predict the optimal or suboptimal secondary structures [2, 3, 4]. In principle, dynamic programming algorithms can predict optimal RNA secondary structure with pseudoknots. But in practice, dynamic programming algorithms can be applied to small RNAs only, and cannot be applied to moderate or large RNAs due to computational complexity of the algorithm.

We have developed a genetic algorithm for predicting RNA secondary structure with pseudoknots. A genetic algorithm (GA) is a non-deterministic optimization procedure, derived from the concept of biological evolution. A genetic algorithm can solve an optimization problem, but it cannot guarantee to find optimal result (see references $[5,6,7,8,9,10]$ for previous works on predicting RNA structure using a

\footnotetext{
* To whom correspondence should be addressed. email: khan@inha .ac.kr
} 
genetic algorithm). To assess the optimality of the predictions by our genetic algorithm, we used thermodynamic free energy as well as the number of base pairs of the predicted structures as fitness functions of the genetic algorithm.

As for the organization of this paper, section 2 describes a genetic algorithm we developed for predicting RNA structure with pseudoknots. Section 3 discusses the predicted RNA structures by the genetic algorithm and compares them with those by a dynamic programming algorithm. Analysis of the results and the general lessons learned from this study are summarized in the final, Conclusion section.

\section{Genetic Algorithm}

For RNA structure prediction, we used a steady-state genetic algorithm because its predictions are known better than others from our previous study [15]. After parsing an RNA sequence, all possible stems were identified as the initial populations of a stem pool. Thermodynamic free energy and the weighted value of the number of base pairs were used as fitness functions. We calculated the value of fitness function in each structure of initial populations and evolved using a genetic operator. RNA structure is represented in a binary string genome, as shown in Fig. 1. As a terminal condition we used both the number of generations and the convergence of each generation.

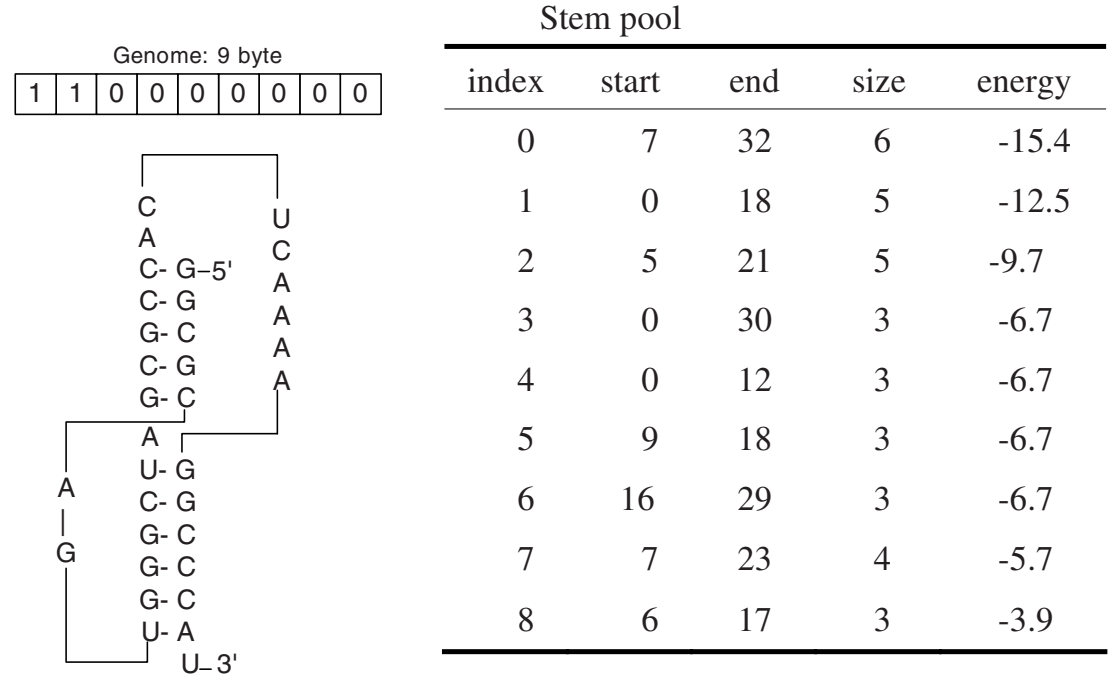

Fig. 1. Example of the representation of the pseudoknot of MMTV RNA in genome

\subsection{Stem Pool Generation}

We generate 3 stem pools after reading a RNA sequence. First we generate all possible stem lists using a covariation matrix. We consider a stem as having minimum 3 
base pairs. We calculate the stacking energy of each stem in stem lists. We sort the stem lists in increasing order of their energy values. This is the first stem pool - what we call "fully zipped stem pool". We then remove consecutive wobble pairs at both ends of a stem since these wobble pairs are not stable enough. After removing all consecutive wobble pairs of each stem at both ends of a stem, we recalculate the stacking energy and removed irregular stems. This is the second stem pool - what we call "partially zipped stem pool". Finally we generate a pseudoknot stem pool by finding all possible pairs of stems that can form a pseudoknot. At this stage, we consider the number of connecting loops and the size of pseudoknot stems only.

These three stem pools can be selectively used in generating initial populations. The purpose of building the partially zipped stem pool is to consider all possible partially zipped stems. The pseudoknot stem pool is for predicting RNA structures with pseudoknots. Without the pseudoknot stem pool, the genetic algorithm predicts secondary structures only.

\subsection{Initial Population}

We generate structures that include every stem in the stem pools. As mentioned above, the three stem pools can be used selectively. After choosing a stem from stem pools, we insert all other stems that can coexist with the chosen stem topologically. If we use the pseudoknot stem pool, we first select 2 stems from the pseudoknot stem pool, and insert all possible stems in other stem pools. In topology test, we check overlapping relation of stems and the type of a pseudoknot. To calculate the free energy of a H-type pseudoknot we apply the known energy model. For pseudoknots of other types, we only check the type of the pseudoknots and use topology test in calculating the free energy of RNA structures. In the example of topology test shown in Fig. 2, cases of (A) and (C) are considered to fail the test.

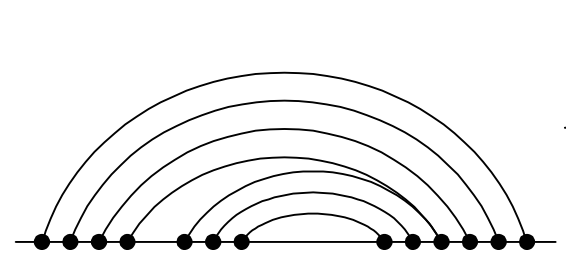

(A) Overlapping stems

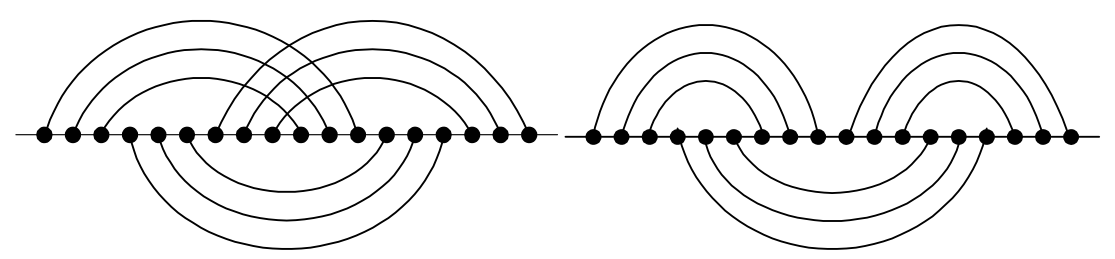

(C) Complex pseudoknot

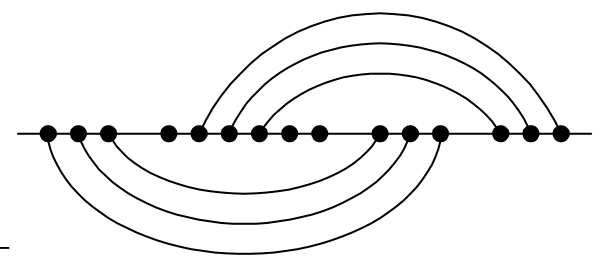

(B) H-type pseudoknot

Fig. 2. Topology test of initial population 


\section{Method and Results}

The genetic algorithm was implemented using C++ builder 5.0 of Inprise Company on $1.61 \mathrm{GHz}$ Pentium $4 \mathrm{PC}$ with $256 \mathrm{MB}$ memory. To analyze the optimality of the genetic algorithm, we compared its predictions to the optimal structures predicted by a dynamic programming algorithm [4].

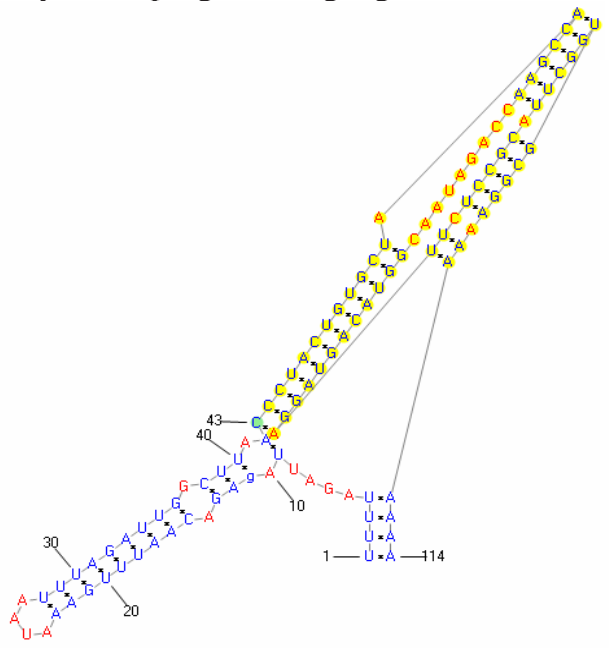

(A)

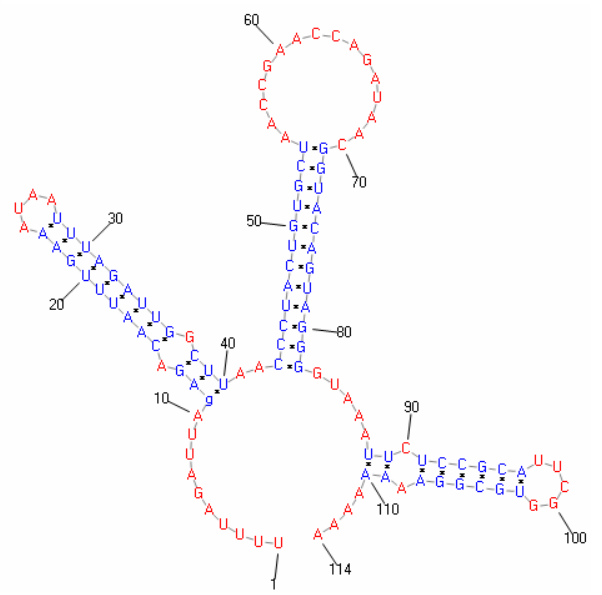

(B)

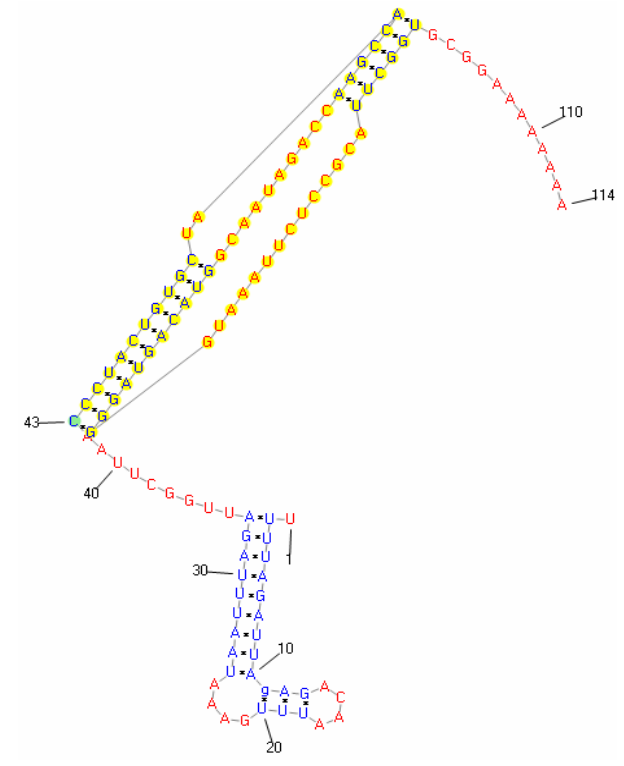

(C)

Fig. 3. (A) known structure of Coxsackie B3 virus. (B) structure predicted by the dynamic programming algorithm. (C) structure predicted by the genetic algorithm using thermodynamic free energy as the fitness function and the partially zipped stem pool and pseudoknot stem pool. 
Since the genetic algorithm is nondeterministic by its nature, we ran the algorithm many times on a same test case. The mean, minimum, maximum and mode values of its predictions were used in comparison with the predictions by a dynamic programming algorithm. Different initial populations were tried using the partially zipped stem pool and fully zipped stem pool.

Fig. 3A shows the known structure Coxsackie B3 virus [11], visualized by PseudoViewer [14]. The structure has a single pseudoknot of nonclassical type. Fig. 3B is the structure predicted by a dynamic programming algorithm. It does not include a pseudoknot. Fig. 3C is the structure predicted by our genetic algorithm using thermodynamic free energy as the fitness function and the partially zipped stem pool and pseudoknot stem pool for initial population.

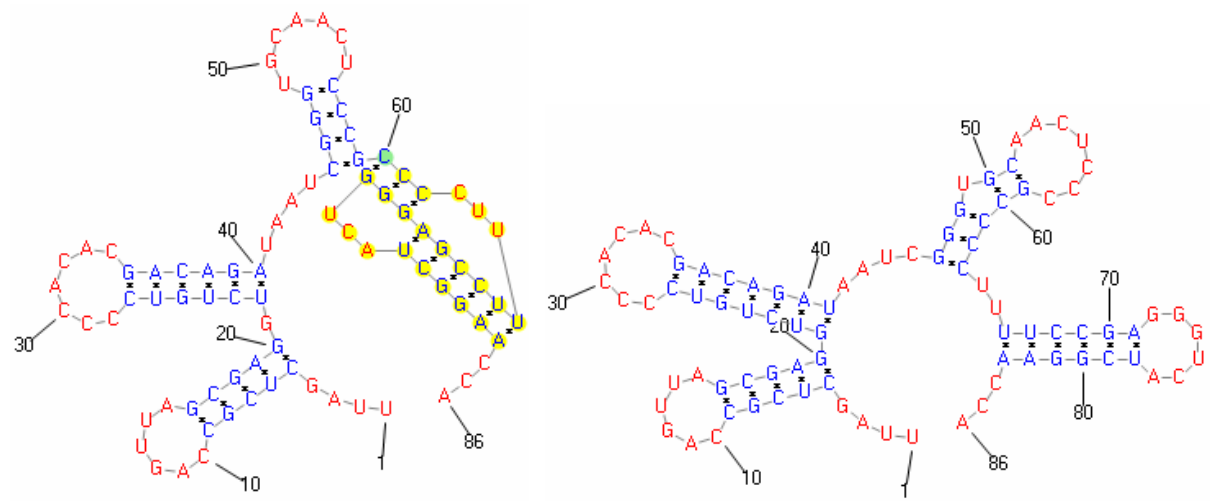

(A)

(B)

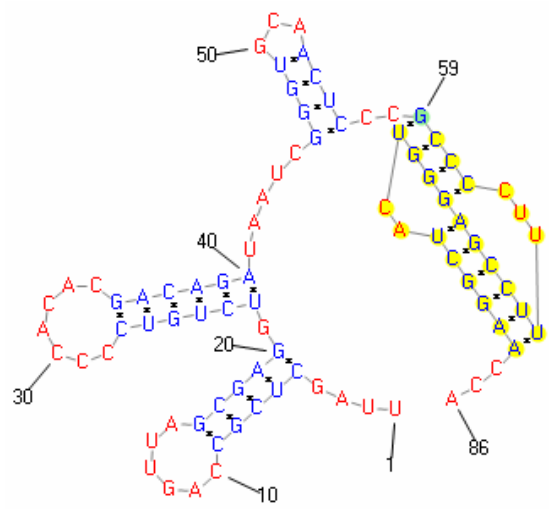

(C)

Fig. 4. (A) known structure of TYMV RNA. (B) structure predicted by the dynamic programming algorithm. (C) structure predicted by the genetic algorithm with thermodynamic free energy as the fitness function and the partially zipped and fully zipped stem pools. 
Figure 4A shows the known structure of TYMV RNA [12], and Fig. 4B is the structure predicted by a dynamic programming algorithm. It does not include a pseudoknot. Figure $4 \mathrm{C}$ is the structure predicted by the genetic algorithm with thermodynamic free energy as the fitness function and the partially zipped stem pool and fully zipped stem pool for initial population. The structure predicted by the genetic algorithm is topologically similar to the known structure in Fig. 4A When the genetic algorithm runs with the fully zipped stem pool and the pseudoknot stem pool, its prediction becomes identical to the known structure.

Table 1 summarizes the analysis of the structures of TYMV and Coxsackie B3 virus predicted by two methods. The two methods were compared with respect to two measures: number of base pairs and free energy of the predicted structures. The exact free energy could not be computed for the known structure of Coxsackie since its pseudoknot structure is not a typical H-type. Although the dynamic programming algorithm predicted the structure with the smallest free energy value, its predictions did not include a pseudoknot at all. The genetic algorithm predicted suboptimal structures in terms of free energy, but the predicted structures are closer to known structures because they contain a pseudoknot.

Table 1. Comparison of predicted structures by a dynamic programming algorithm and a genetic algorithm. Free energy cannot be computed for the known structure of coxsackie

\begin{tabular}{ccccc}
\hline \multirow{2}{*}{ RNA } & measure & $\begin{array}{c}\text { known } \\
\text { structure }\end{array}$ & $\begin{array}{c}\text { prediction by } \\
\text { dynamic } \\
\text { programming }\end{array}$ & $\begin{array}{c}\text { prediction by } \\
\text { GA }\end{array}$ \\
\hline \multirow{2}{*}{ TYMV } & \# base pairs & 24.0 & 23.0 & 25.0 \\
& free energy & -24.8 & -29.6 & -24.1 \\
\multirow{2}{*}{ Coxsackie } & \# base pairs & 41.0 & 32.0 & 29.0 \\
& free energy & - & -34.4 & -30.7 \\
\hline
\end{tabular}

Table 2. Statistical values of the predictions by the genetic algorithm

\begin{tabular}{llrr}
\hline \multicolumn{1}{c}{ stem pool } & \multicolumn{1}{c}{ measure } & Coxsackie RNA & TYMV RNA \\
\hline & \# stems & 132 & 62 \\
& \# predicted structures & 1 & 11 \\
partially zipped stem pool & maximum energy & -30.7 & -24.8 \\
pseudoknot stem pool & minimum energy & -30.7 & -13.0 \\
& mean energy & -30.7 & -20.4 \\
& mode energy & -30.7 & -24.8 \\
\hline & \# stems & 229 & 113 \\
partially zipped stem pool & \# predicted structures & 6 & 14 \\
fully zipped stem pool & maximum energy & -45.6 & -26.2 \\
pseudoknot stem pool & minimum energy & -25.7 & -18.1 \\
& mean energy & -29.9 & -22.9 \\
& mode energy & -30.7 & -24.1 \\
\hline
\end{tabular}


Table 2 summarizes the statistical values of the predictions by several runs of the genetic algorithm. In Table 2, we compare the predictions by the genetic algorithm with the partially zipped stem pool alone with those with both the partially zipped stem pool and the fully zipped stem pool. The pseudoknot stem pool was used commonly in both cases. By using the fully zipped stem pool additionally, we could predict many suboptimal structures, and also more stable structures. But as the size of stem pools increases, the genetic algorithm takes more time.

The execution time of the genetic algorithm depends on its termination condition: the number of generations or the convergence of a generation. The convergence of a generation was used to predict the structures shown earlier. Table 3 shows the effect of changing the number of generations when running the genetic algorithm.

Table 3. The effect of changing the number of generations when running the genetic algorithm

\begin{tabular}{cccccc}
\hline $\begin{array}{c}\# \\
\text { generations }\end{array}$ & $\begin{array}{c}\text { \# predicted } \\
\text { structures }\end{array}$ & $\begin{array}{c}\text { maximum } \\
\text { energy }\end{array}$ & $\begin{array}{c}\text { minimum } \\
\text { energy }\end{array}$ & $\begin{array}{c}\text { mean } \\
\text { energy }\end{array}$ & $\begin{array}{c}\text { mode } \\
\text { energy }\end{array}$ \\
\hline 50 & 8 & -24.8 & -13.9 & -21.51 & -24.8 \\
150 & 9 & -24.8 & -13.9 & -21.39 & -24.8 \\
250 & 6 & -24.8 & -13.9 & -22.41 & -24.8 \\
\hline
\end{tabular}

We predicted the TYMV RNA using different numbers of generations. We found that there is little difference between the case using the convergence and that using the number of generations and that it converged to the most stable structure early.

We also predicted the RNA structures with long sequences. Longer sequences need more execution time. The genetic algorithm takes very long when the sequence length exceeds 300 bases. Fig. 5 and Fig. 6 show the structures of DIGIR1 RNA and TMV RNA, respectively $[1,13]$. Fig. 5A is the known structure of DIGIR1 RNA and Fig. 5B is the predicted structure by the genetic algorithm. Fig. 6A is the known structure of TMV RNA with 4 H-type pseudoknots and 6 stems outside the pseudoknots. The genetic algorithm predicted a similar structure to the known structure with free energy as its fitness function. Both the partially zipped stem pool and the pseudoknot stem pool were used, but the fully zipped stem pool was not used due to the size of the RNA sequence. The algorithm terminated evolving process using the convergence of populations. The structures shown in Fig. 6B-D were obtained by the genetic algorithm with different fitness functions. The structure shown in Fig. $6 \mathrm{C}$ was predicted by the genetic algorithm with the weighted value of base pairs as the fitness function instead of energy value. Both structures of Fig. 6B and Fig. 6D were predicted by the genetic algorithm with free energy as the fitness function. Fig. 6B had a higher energy value (i.e., less stable) than the structure of Fig. 6D. The structure of Fig. 6D is the most similar to the known structure. The known structure of TMV has 14 stems, but 2 of them could not be generated at initial stage. The structure shown in Fig. 6C was obtained with the weighted value of base pairs, which is a rough approximation of free energy, but is similar to the known structure in Fig. 6A. For the structure with nonclassical pseudoknots, the genetic 
algorithm performs better with the weighted value of base pairs than with free energy since the exact energy model is not available for nonclassical pseudoknots.

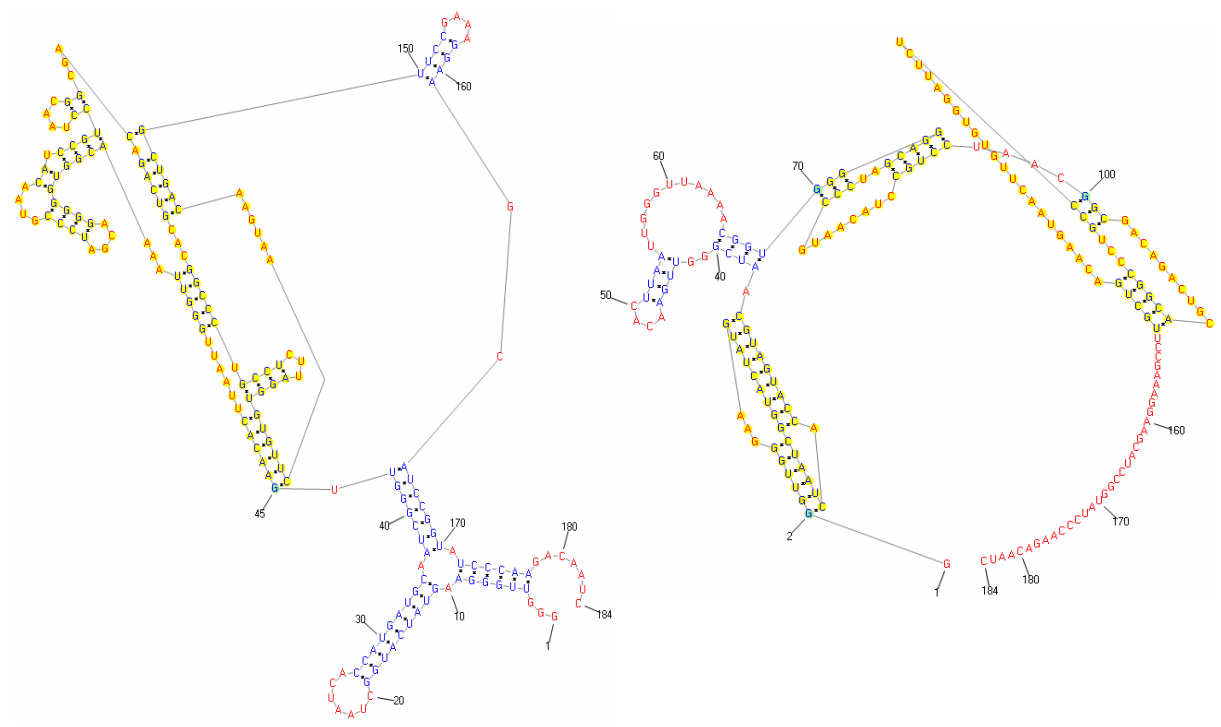

(A)

(B)

Fig. 5. (A) known structure of DIGIR1 RNA. (B) predicted structure by the genetic algorithm.

\section{Conclusion}

We developed a genetic algorithm for predicting the RNA structures with pseudoknots, and compared the results from using different stem pools and fitness functions. We also compared the structures predicted by the genetic algorithm to the structures predicted by a dynamic programming algorithm.

The known RNA structures are often the suboptimal structures in terms of free energy, and therefore the optimal structures predicted by a dynamic programming algorithm may not correspond to the known structures. Genetic algorithms do not guarantee to find optimal structures but often predict better than dynamic programming algorithms. The difficulties of predicting RNA structures using a genetic algorithm come from several things. First, there exist stems of irregular types in natural RNAs, but all those irregular types cannot be considered during the population generation stage. This means that the quality of the prediction depends on the stem pools used. Second, no energy model is not available for nonclassical pseudoknots, and the energy model for H-type pseudoknot is not accurate, either. Therefore, simple and rough fitness criteria such as the weighted sum of base pairs can be a good fitness function when the exact energy model associated with nonclassical pseudoknots is not available. 


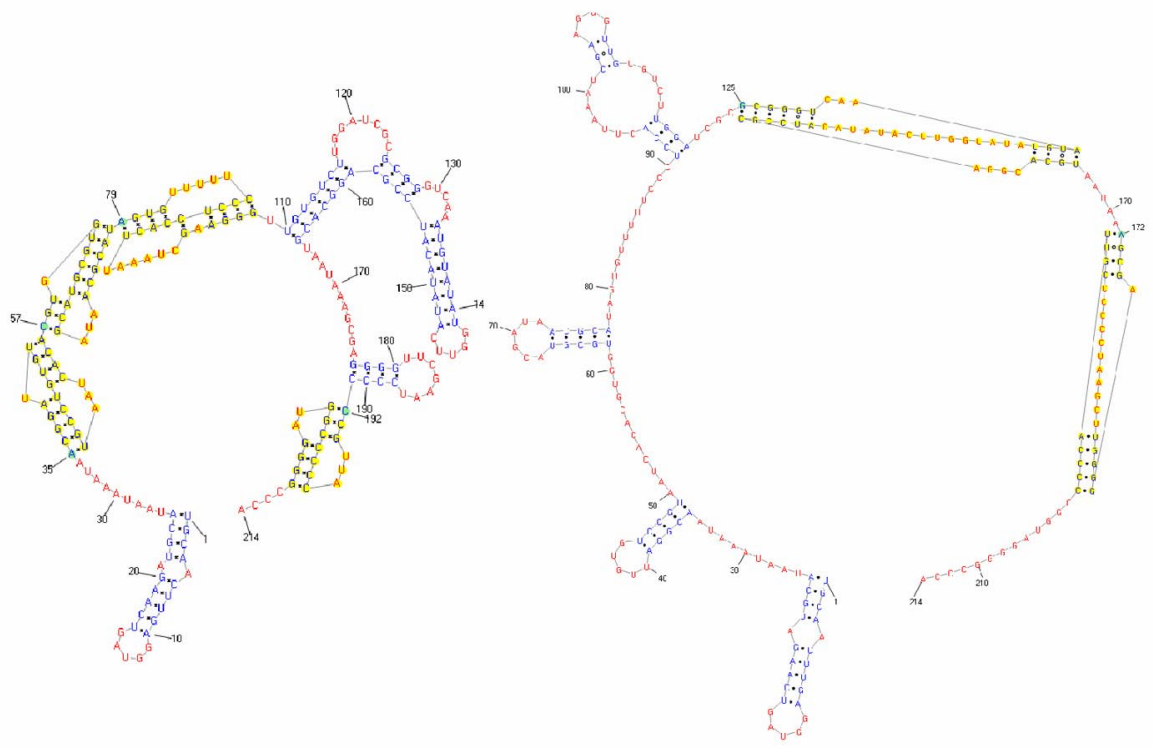

(A)

(B)

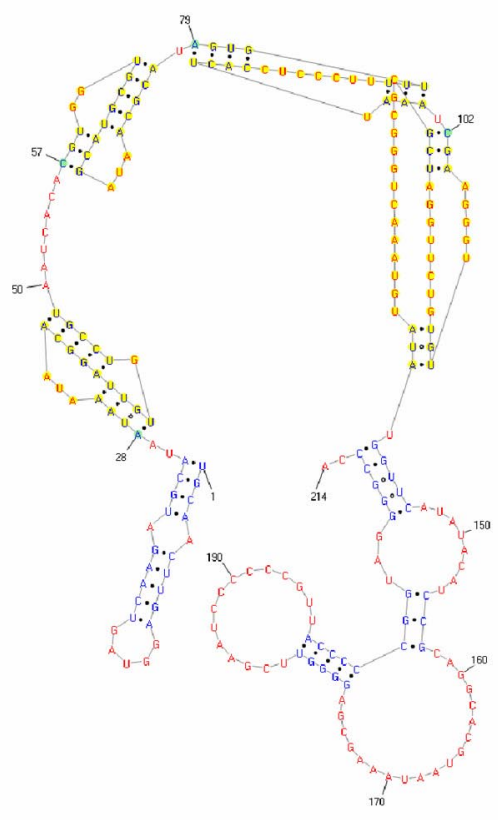

(C)

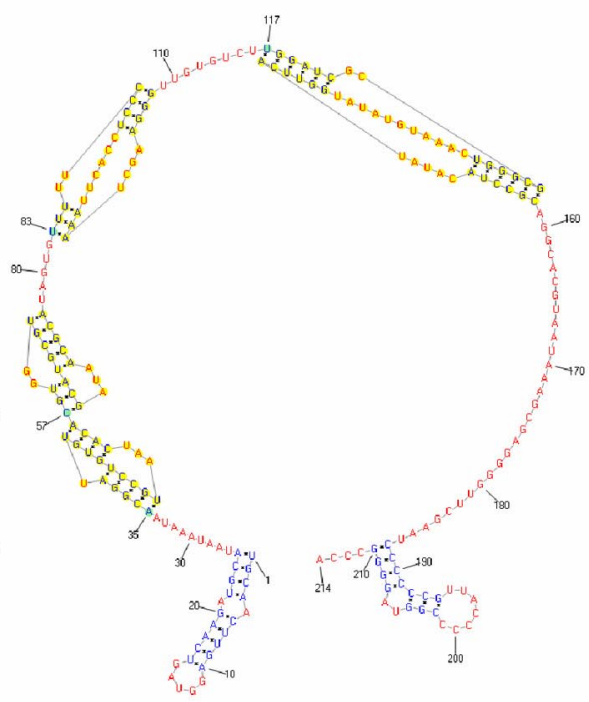

(D)

Fig. 6. (A) known structure of TMV RNA with 4 H-type pseudoknots. (B) structure predicted by the genetic algorithm with the weighted sum of base pairs as the fitness function. Structures of (B) and (D) were predicted by the genetic algorithm with free energy as the fitness function. 
Acknowledgement. This work has been supported by the Korea Science and Engineering Foundation (KOSEF) under grant R05-2001-000-01037-0.

\section{References}

1. Chen, J.-H., Le, S.-Y., and Maizel, J. V.: A procedure for RNA pseudoknot prediction. Computer Applications in the Biosciences 8 (1992) 243-248

2. Abrahams, J. P., van den Berg, M., van Batenburg, E. and Pleij, C.: Prediction of RNA secondary structure, including pseudoknotting, by computer simulation. Nucleic Acids Res. 18 (1990) 3035-3044

3. Akutsu, T.: Dynamic programming algorithm for RNA secondary structure prediction with pseudoknots. Discrete Applied Mathematics 104 (2000) 45-62

4. Rivas, E. and Eddy S. R.: A dynamic programming algorithm for RNA structure prediction including pseudoknots. Journal of Molecular Biology 285 (1999) 2053-2068

5. Gultyaev, A. P., van Batenburg, F. H. D. and Pleij, C. W. A.: The computer simulation of RNA folding pathways using a genetic algorithm. Journal of Molecular Biology. 250 (1995) 37-51

6. Shapiro, B. A. and Wu, J. C.: Predicting RNA H-Type pseudoknots with the massively parallel genetic algorithm. Computer Applications in the Biosciences 13 (1997) 459-471

7. Shapiro, B. A. and Wu, J. C.: An annealing mutation operator in the genetic algorithms for RNA folding. Computer Applications in the Biosciences 12 (1996) 171-180

8. Shapiro, B. A., Wu, J. C., Bengali, D. and Potts, M. J.: The massively parallel genetic algorithm for RNA folding: MIMD implementation and population variation. Bioinformatics 17 (2001) 137-148

9. Benedetti, G. and Morosetti, S.: A genetic algorithm to search for optimal and suboptimal RNA secondary structures. Biophysical Chemistry 55 (1995) 253-259

10. Shapiro, B. A. and Navetta, J.: A massively parallel genetic algorithm for RNA secondary structure prediction. Journal of Supercomputing 8 (1994) 195-207

11. Deiman, B. A. and Pleij, C. W. A.: A vital feature in viral RNA. Seminars in Virology 8 (1997) 166-175

12. Hilbers, C. W., Michiels, P. J. A. and Heus, H. A.: New Developments in structure determination of pseudoknots. Biopolymers 48 (1998) 137-153

13. Einvik, C., Nielsen H., Nour, R. and Johansen, S.: Flanking sequences with an essential role in hydrolysis of a self-cleaving group 1-like ribozyme. Nucleic Acids Res. 28 (2000) 2194-2200

14. Han, K., Lee, Y., Kim, W.: PseudoViewer: automatic visualization of RNA pseudoknots, Bioinformatics 18 (2002) S321-S328

15. Lee, D., Han, K.: Prediction of RNA pseudoknots-comparative study of genetic algorithms. Genome Informatics 13 (2002) 414-415 